\title{
LA EFICIENCIA TÉCNICA DE LA INDUSTRIA AUTOMOTRIZ EN MÉXICO, I988-2008
}

\author{
Eliseo Díaz González, Jairo César López Zepeda ${ }^{\mathrm{a}}$ \\ y Rafael Garduño Rivera ${ }^{\mathrm{b}}$
}

Fecha de recepción: 9 de enero de 2019. Fecha de aceptación: 4 de junio de 2019.

http://dx.doi.org/10.22201/iiec.20078951e.2019.199.68192

Resumen. El presente trabajo evalúa el impacto de la especialización productiva en la eficiencia técnica de la industria automotriz en México (1988-2008), utilizando el método de frontera de posibilidades de producción en escala regional, y considerando su localización regional. Para ese propósito, se estimó un índice de especialización regional del sector y un índice de eficiencia técnica para la industria automotriz utilizando el modelo de fronteras estocásticas (Battese y Coelli, 1995). Los hallazgos obtenidos sugieren que la especialización tiene impacto positivo en la eficiencia productiva en las unidades de análisis, además, se muestra que la escolaridad y la localización de las plantas automotrices en la región norte y centro del país contribuyen a disminuir los niveles de ineficiencia productiva.

Palabras clave: industria automotriz; especialización productiva; eficiencia técnica; clústeres automotrices; fronteras estocásticas.

Clasificación JEL: C33; L62; L92; O14.

\section{TeChNiCAl EFFiciency In the MeXican} AUTOMOTIVE INDUSTRY, I 988-2008

\begin{abstract}
This work evaluates the impact of productive specialization on the technical efficiency of the automotive industry in Mexico (1988-2008), using the productionpossibility frontier method on a regional scale and considering its regional localization. To this end, an index of regional specialization in said sector was calculated, in addition to a technical efficiency index for the automotive industry using the stochastic frontier model (Battese and Coelli, 1995). The findings that were obtained suggest that specialization has a positive impact on productive efficiency in the units of analysis, and further, demonstrate that education levels and the localization of automotive plants in the northern and central regions of the country contribute to decreasing levels of productive inefficiency.
\end{abstract}

Key Words: automotive industry; productive specialization; technical efficiency; automotive clusters; stochastic frontier.

a El Colegio de la Frontera Norte (COLef), México; ${ }^{b}$ Universidad Panamericana, sede Aguascalientes, México. Correos electrónicos: ediaz@colef.mx; jairomea2016@colef.mx y rgardunor@up.edu.mx, respectivamente. 


\section{INTRODUCCIÓN}

La industria automotriz en México surge en la década de los veinte del siglo xx con la instalación de las primeras líneas de ensamblaje de la marca Ford, iniciando así una de las principales industrias manufactureras en México (Covarrubias, 2014). En los años ochenta, gracias a la apertura económica y la cuenta de capitales, la ampliación de la propiedad y el control por parte de extranjeros, la industria se dirige ya no sólo a abastecer el mercado interno, sino que se orienta también al mercado externo. Este proceso se consolida con la entrada en vigor del Tratado de Libre Comercio de América del Norte (TLCAN) en 1994, que incluyó nuevas reglas, la reducción progresiva de tarifas arancelarias y del componente nacional en la producción dirigida a la exportación. De esta manera, la expansión de la industria automotriz en México y su consolidación es resultado de la combinación de una política industrial interna y el proceso de globalización económica (Miranda, 2007), origen de la apertura de la economía mexicana. En la actualidad, la industria automotriz se ha consolidado como una de las principales industrias manufactureras en México.

El sector automotriz se ha estudiado en México desde distintas perspectivas, como es la histórica (Miranda, 2007); laboral (Dombois, 1985; Arteaga, 2003; Covarrubias, 2014) y regional (Unger y Chico, 2004; Chávez-Martín del Campo y Fonseca, 2013), entre otras. Sin embargo, en eficiencia técnica los trabajos están focalizados en su mayoría sobre el sector manufacturero y en entidades federativas. El presente análisis se basa en un enfoque microeconómico con mayor nivel de desagregación geográfica, siguiendo la metodología de fronteras estocásticas (SFA, por sus siglas en inglés). Se estima la eficiencia técnica con una metodología paramétrica, es decir, se utiliza una función de producción tipo Cobb-Douglas con dos y tres factores productivos, pero se incluyen además variables de control como escolaridad, especialización productiva, regiones geográficas o acuerdos comerciales, para evaluar su impacto en la eficiencia técnica. De esta forma, el análisis propone explicar la eficiencia técnica del sector automotriz incluyendo la especialización productiva y la liberalización económica.

Se utiliza un segundo modelo para medir los determinantes de la ineficiencia técnica, donde el indicador de ineficiencia aparece explicado por seis variables; algunas como el índice de especialización, incluidas también en la parte determinista de la ecuación. En el modelo de datos de panel utilizado, las secciones cruzadas son: regiones -zonas metropolitanas o municipios del 
país- con variables temporales, es decir, unidades de tiempo y variables de producción, empleo, etcétera, de la industria automotriz. Todos los datos provienen de los censos económicos nacionales.

La hipótesis de trabajo sostiene que el desarrollo reciente de la industria automotriz ha creado cierta especialización productiva en regiones que concentran la exportación de bienes automotrices, y que estas regiones conforman la frontera de posibilidades de producción de la industria automotriz en México. Así, se pretende dar respuesta a preguntas que se desprenden de esta hipótesis: indagar acerca de cuáles son las unidades de análisis más especializadas en la industria automotriz; cuál es el efecto de la especialización en la eficiencia técnica de la industria automotriz; si en verdad el TLCAN contribuyó a reducir la ineficiencia productiva en México; si existe una diferencia significativa en la eficiencia técnica en las regiones de producción automotriz y, finalmente, establecer cuáles son los determinantes de la eficiencia técnica automotriz en las zonas geográficas analizadas.

El documento se estructuró en seis apartados incluyendo esta introducción; el segundo expone el desarrollo de la industria automotriz en México mediante una breve revisión de sus etapas de desarrollo hasta su contexto en la actualidad. El apartado tres contiene la revisión teórica y metodológica utilizada en el análisis; ahí se expone una revisión teórica de especialización y localización (Krugman, 1991; Goldstein y Gronberg, 1984; Eberts y McMillen, 1999; Venables, 1996; Fujita et al.,1999). Después, en el cuarto apartado, se presenta la teoría microeconómica relacionada con la eficiencia técnica y los principales métodos de estimación (análisis envolvente de datos y SFA), además de las principales funciones de producción para el caso de la estimación paramétrica (SFA). También son expuestos los estudios sobre eficiencia técnica en México, seguido del modelo empírico estimado. Asimismo, los datos y la estadística descriptiva de las variables empleadas en el modelo empírico. En el quinto apartado, se presentan los resultados obtenidos del modelo de eficiencia técnica estimado. El último apartado presenta las conclusiones.

\section{LA INDUSTRIA AUTOMOTRIZ EN MÉXICO}

El sector automotriz es el principal generador de divisas con un superávit comercial mayor a US\$71 billones de dólares en 2017 (INEGI, 2018), con exportaciones que representan $31 \%$ del total de las exportaciones manufactureras del país, con un valor de US $\$ 126.7$ billones. También es uno de los mayores receptores de Inversión Extranjera Directa (IED), entre el 2000 y el 
2017 captando alrededor de US\$60.6 billones, por encima de la banca múltiple (56.1 billones) y la industria de las bebidas (39.3 billones) (AMIA, 2018). México es el cuarto país en producción de vehículos, con una participación de 7.6\% del total de las exportaciones de automóviles, sólo por debajo de Alemania, Japón y Estados Unidos de América con 17.8, 9.9 y 9.4\% de participación, respectivamente (INEGI, 2018).

Esta industria evoluciona mediante la instalación de plantas armadoras a lo largo del país en distintos periodos de tiempo, diversos contextos económicos internacionales y políticas industriales particulares. Inicia con la instalación de la primera planta Ford en 1925, pasando por distintas etapas de desarrollo hasta su consolidación en el periodo de liberalización económica mexicana (Miranda, 2007). Entre 1962 y 1976 se emite el primer decreto automotriz que restringía las importaciones de vehículos y de autopartes, y entonces fijaba en $60 \%$ el contenido nacional en vehículos terminados y limitaba a $40 \%$ la participación de IED en plantas armadoras, además de establecer controles de precios. Es bajo este panorama que arriban importantes marcas automotrices como Volkswagen en Puebla, General Motors y Chrysler en el Estado de México, y Nissan en Morelos.

Ante un creciente déficit en la balanza de pagos -derivado de la crisis del petróleo en los ańos setenta-, en el periodo de 1977-1989 la regulación fue más flexible. Un decreto de 1977 propone convertir a México en un país exportador competitivo, reduciendo a $50 \%$ el contenido nacional de la producción local destinada a exportación, manteniendo la protección en relación con la participación en la inversión.

La competencia que representaban los vehículos japoneses en el mercado estadounidense fue un factor que motivó a la industria norteamericana a invertir en México, buscando disminuir costos de producción y hacer frente a la nueva competencia. De esta forma, se instalan plantas de ensamblaje y producción de motores de compañías norteamericanas en el norte del país: General Motors y Chrysler en Ramos Arizpe (1981), Ford en Chihuahua y en Sonora en 1983 y 1986, respectivamente. En 1989 se emite un decreto con una orientación de liberalización económica que busca modernizar la industria, aumentar su eficiencia y productividad. La nueva regulación permitía la importación de vehículos nuevos, ofrecía concesiones fiscales equivalentes al $30 \%$ de inversión y la disminución del contenido nacional de 50 a 36\% para los vehículos exportados.

En 1994 el TLCAN, en conjunto con otras medidas complementarias, llevaron a consolidar la liberalización económica en 2004, que eliminó todo rasgo de proteccionismo. Las nuevas reglas comerciales incluían reducciones 
progresivas de aranceles y de composición de contenido nacional de autos de exportación hasta su completa eliminación en 2004. ${ }^{1}$ Esto trajo consigo nuevas inversiones en el norte y centro del país. Se establecen Mercedes Benz en Nuevo León (1994), Honda en Jalisco (1994), General Motors en Guanajuato (1995) como resultado de la relocalización desde la Ciudad de México; Chrysler en Coahuila (1995), BMw y volvo en el Estado de México en 1995 y 2000 , respectivamente. En suma, se conforma la planta industrial automotriz que se conoce hasta hoy, misma que alcanza cifras récord en inversiones, valor de exportaciones y que convierte a México, como se mencionó, en un importante exportador de vehículos.

\section{LOCALIZACIÓN Y ESPECIALIZACIÓN PRODUCTIVA}

En México, las manufacturas automotrices se concentran en regiones específicas del territorio nacional (Mendoza, 2003). El concepto de aglomeración se vincula a nuevos enfoques de la geografía económica que destacan el potencial competitivo asociado con la estrecha relación entre la demanda y la oferta de los grupos regionales de industrias aliadas. Tratan de explicar los factores que determinan el desarrollo industrial en un espacio geográfico determinado (Porter, 1982; Krugman y Venables, 1995). Para algunos autores, los mercados locales especializados en mano de obra o productos intermedios son factores que por lo general detonan la proximidad productiva (Driffield y Munday, 2001). La concentración industrial presenta externalidades positivas asociadas a un proceso de efectos alternos, derivados, por ejemplo, de la innovación tecnológica o bien de la organización industrial (Arrow, 1962; Romer, 1986; Marshall, 1920; Jacobs, 1969).

Las principales ventajas económicas que las empresas o industrias pueden aprovechar, dada su localización (Eberts y McMillen, 1999), se relacionan con las economías de escala al interior de la firma; economías de localización relacionadas con la concentración de una misma industria en una zona geográfica, y economías de urbanización, relacionadas con factores no comunes de la actividad productiva como la infraestructura pública.

La concentración industrial es resultado de la interacción en la demanda industrial de firmas o empresas que deciden establecerse muy próximas una de otra para minimizar costos fijos y de transporte (Krugman, 1981). Este

1 La composición de contenido nacional para autos de exportación en 1993 fue de 36\%; en 1998 de $29 \%$, y en 2004 de $0 \%$. 
modelo básico de economías de escala al interior de la firma sigue al modelo de comercio internacional desarrollado por Krugman (1980), aunque las unidades de análisis ahora no son naciones sino regiones. En otro modelo, la concentración deriva de la especialización de bienes intermedios y resulta de la minimización de costos y la interacción entre firmas por el lado de la demanda (Venables, 1996).

Los spillovers de conocimiento tienen limitaciones geográficas que no pueden ser medibles, dado que no dejan ningún tipo de rastro tangible que pueda ser cuantificable (Krugman, 1991). Otros trabajos tratan de plantear soluciones al problema de la cuantificación del conocimiento en la geografía económica (Jaffe, 1989; Feldman, 1994; Audretsch y Feldman, 1996).

Este tipo de economías ofrecen servicios especializados -infraestructura pública como carreteras, parques industriales, instalaciones de energía, seguridad, etcétera- en grandes áreas urbanas que, por tanto, no se forman en zonas de menor tamaño (Goldstein y Gronberg, 1984). Las metodologías más desarrolladas para incorporar la heterogeneidad entre firmas son las que se basan en la estimación de fronteras de producción (e.g. Christensen et al., 1973; Aigner et al., 1977; Battese y Coelli, 1995; Cullinane et al., 2006; Driffield y Munday, 2001; Kirkley et al., 1995; Reinhard et al., 1999; Seyoum et al., 1998; Tovar y Martín-Cejas, 2010). Sin embargo, son todavía escasos los estudios empíricos de fronteras de producción orientados al análisis del vínculo entre especialización y eficiencia productiva (e.g. Álvarez-P. et al., 2017; Bannister y Stolp, 1995; Acevedo y Ramírez, 2005).

\section{EFICIENCIA TÉCNICA Y MÉTODOS DE ESTIMACIÓN}

Una definición de eficiencia técnica sostiene que, bajo un conjunto de posibilidades de producción, un vector de entrada y otro de salida son técnicamente eficientes si no existe otro vector de entrada que utilice una menor cantidad de insumos para producir la misma cantidad de salida (Koopmans, 1951).

Se tienen dos categorías de eficiencia: la técnica y la asignativa (Álvarez-P., 2013). La eficiencia técnica se refiere al aprovechamiento de los recursos en términos de insumos, es decir, la relación existente entre los insumos productivos utilizados y el producto terminado. La eficiencia técnica, por tanto, es la capacidad de producir bienes con la menor cantidad de insumos disponibles (Farrell, 1957). La eficiencia asignativa se refiere a la minimización de costos y 
maximización de beneficios, obedece a la asignación de precios por oferta y demanda. Dado que el presente trabajo se refiere al adecuado aprovechamiento de los insumos productivos, entra dentro del concepto de eficiencia técnica.

Existen múltiples formas de estimar la eficiencia técnica, cada una de ellas con argumentos técnicos y metodológicos que ofrecen ventajas y desventajas (Álvarez-P., 2013). En este trabajo se seleccionó el método de sFA como la metodología de estimación, pues es la que se ajusta mejor a la solución del problema de investigación planteado. El análisis de SFA es una técnica de estimación paramétrica, que involucra una función de producción que puede tener distintas variantes al incorporar a esta función un error aleatorio, que se denomina frontera estocástica. Este término de error contiene dos componentes, uno que mide el efecto aleatorio y otro la ineficiencia. El modelo básico original (Aigner et al., 1977) presenta la siguiente estructura:

$y_{i}=f\left(x_{i} ; \beta\right)+\varepsilon_{i}, i=1, \ldots, N$

Donde $y_{i}$ es la máxima producción posible de la unidad $i$ dado un vector de insumos $x_{i}$, $\beta$ es un parámetro por estimar y $\varepsilon_{i} \leq 0$ es el término de error, el cual tiene el componente aleatorio y de ineficiencia como es mostrado en la siguiente ecuación:

$\varepsilon_{i}=v_{i}+u_{i}, \mathrm{i}=1, \ldots, N$

Por un lado, $v_{i}$ que es el error idiosincrático que se asume independiente e idénticamente distribuido con $N(0, \sigma 2)$, por otro lado $u_{i}$ es el error que mide la ineficiencia, se asume distribuido independiente de $v_{i}$ y presenta una distribución normal truncada positiva, de tal manera que se satisface que $u_{i} \leq 0$. Es decir, valores mayores a cero en $u_{i}$ implican unidades de observación ineficientes, mientras que valores iguales a cero implican unidades sobre la frontera eficiente.

Este modelo no incorpora la especificación de variables explicativas del término de ineficiencia y era estimable sólo para datos de corte transversal (Aigner et al., 1977). Posteriormente, otros autores desarrollaron uno de los primeros trabajos que incorpora variables explicativas al modelo de eficiencia, modelaron la eficiencia técnica de establecimientos de tejido en Indonesia a partir del tipo de propiedad, edad y establecimiento de unidades de análisis, sin embargo, no se incluía temporalidad en la eficiencia, es decir, no permitía variaciones de tiempo del término aleatorio (Pitt y Lee, 1981). 


\section{Modelo empírico}

La técnica de estimación planteada para este análisis ha sido desarrollada por otros autores como Battese y Coelli (1995) dado que se cuenta con datos en forma de panel, y se utiliza la función de producción especificada en otro trabajo de los años setenta (Aigner et al., 1977) en la forma que se muestra a continuación:

Función de producción estocástica tipo Cobb-Douglas (1928):

$$
y_{i t}=\left(x_{i t} \beta\right)+\varepsilon ; \varepsilon=V_{i t}-U_{i t}
$$

Error aleatorio asociado con la eficiencia:

$$
\begin{aligned}
& U_{i t}=z_{u} \delta \\
& U_{i t} \sim N+\left(z_{u} \delta, \sigma^{2}{ }_{u}\right)
\end{aligned}
$$

La ecuación general de la sección determinística, la modelación de la frontera de posibilidades de producción sigue una función tipo Cobb-Douglas de la forma:

$$
\begin{aligned}
\ln Y_{i t} & =\beta_{0}+\ln \beta_{1} K+\ln \beta_{2} L+\sum_{j=3} \\
\beta_{j} x_{j i t} & +\varepsilon \\
\varepsilon & =V_{i t}-U_{i t}
\end{aligned}
$$

Donde $i$ indica el área geográfica de análisis (municipios y zonas metropolitanas); $Y, K$ y $L$ son variables que representan la producción automotriz, capital y trabajo, respectivamente, que se detallan más adelante; $x$ es un grupo de control para la estimación de la frontera de posibilidades de producción, $j$ indica el número de variables de control utilizadas, 3 en este caso, o bien $j=3$, que son la especialización productiva, la población y la energía eléctrica. Todas las variables que contienen esta forma funcional son presentadas en logaritmo natural, a excepción del índice de especialización productiva.

La modelación del error $U_{i t}$ relacionado con las variables explicativas de la ineficiencia, se presenta como:

$$
\begin{aligned}
U_{i t}= & \delta_{0}+\delta_{1} e s p+\delta_{2} \text { lnesc }+\delta_{3} t+\delta_{4} t^{2}+\delta_{5} \text { arm }+\delta_{6} \text { cnor } \\
& +\delta_{7} \text { nor }+\delta_{8} \text { cen }+\delta_{9} \text { sur }+\delta_{10} \text { tlcan }+\delta_{11} z m
\end{aligned}
$$


Son 11 variables que modelan la varianza de la ineficiencia o la sección estocástica: esp, referente a la especialización productiva; lnesc, como escolaridad; $t$ indica variaciones no observadas en el tiempo; $t^{2}$ tendencia al cuadrado; arm variable dicotómica que identifica si la unidad de análisis contiene una planta armadora, la inclusión de esta variable se justifica dado el tipo de capital y tecnología implementadas en las plantas armadoras. El hecho de que estas plantas puedan contener una estructura productiva más sofisticada (con mayor tecnología y organización) puede impactar en la eficiencia productiva de las unidades de análisis; cnor, nor, cen, sur, son variables dicotómicas para indicar si las unidades de análisis pertenecen a la región centro norte, norte, centro y sur, respectivamente. El tlcan es una variable instrumental que se introduce para conocer el efecto del TLCAN con el fin de evaluar si existe mejora en la eficiencia productiva automotriz dada la apertura comercial; $z m$ variable dicotómica para indicar si la unidad de observación pertenece o no a una zona metropolitana, esta variable se incluye para evaluar efectos inobservados de economías de urbanización (Goldstein y Gronberg, 1984).

En México y otros países, distintos trabajos han estimado la eficiencia técnica tanto por el método de la envolvente como por SFA, enfocados en particular en el sector manufacturero. Con la metodología del análisis envolvente de datos, destacan los trabajos de Bannister y Stolp (1995), Navarro y Torres (2006), Arellano y Cortés (2010), Montiel (2012), y Becerril et al. (2012). Con el método de sfa los estudios de Becerril et al. (2012), Gutiérrez (2011), Chávez-Martín del Campo y Fonseca (2013); para el caso del potencial de recaudación fiscal de los estados, Peón y Casimiro (2017) y Álvarez-P. et al. (2015).

\section{Eficiencia técnica y fronteras estocásticas}

Se analiza la industria automotriz para el periodo 1994-2009, que incluye los censos económicos 1994, 1999, 2004 y 2009, ${ }^{2}$ en las ramas industriales 3361 (Fabricación de automóviles y camiones); 3362 (Fabricación de carrocerías y remolques); y 3363 (Fabricación de partes para vehículos automotores) del Sistema de Clasificación Industrial de América del Norte (scian). Para 1999 se refiere a la rama 3841 (Industria Automotriz). Dentro de estas ramas son tomadas las variables Valor Agregado, Activos Fijos, Personal Ocupado y Consumo de Energía Eléctrica que se describen más adelante.

2 Se excluyeron los datos del Censo Económico 2014 al no reportar cifras del sector automotriz a nivel municipio incluidos en ediciones anteriores. 
Para la estimación del modelo de eficiencia técnica se utiliza la técnica de datos de panel balanceado con 72 secciones cruzadas: 38 corresponden a zonas metropolitanas y 34 a municipios no agrupados, que alojan actividad automotriz durante el periodo censal descrito. La delimitación de áreas metropolitanas fue tomada del Consejo Nacional de Población (Conapo, 2010) y los municipios del Marco Geoestadístico Nacional del INEGI.

La eficiencia técnica es modelada con base en un grupo de variables que estiman la frontera de posibilidades de producción (sección determinística) y la ineficiencia técnica (sección estocástica). Existe controversia acerca de las variables que deben incluirse en una y otra sección (Álvarez-P. et al., 2017), pero, en general, las variables de control de la frontera pueden ser incluidas también en la sección de ineficiencia, sin embargo, dependerá del caso particular de análisis para considerar cuáles estarán en una u otra sección.

La variable dependiente (y) utilizada para la estimación del modelo de SFA es el valor agregado automotriz, que es deflactado para cada corte de tiempo con el Índice Nacional de Precios al Consumidor (INPC) base 2010. Para la modelación de la sección determinística, la variable fue transformada a logaritmos naturales.

La gráfica 1 muestra que prácticamente en todo el periodo de análisis, el Valor Agregado Automotriz experimentó crecimiento en términos reales. De 1988 a 2008, un lapso de 20 años, la producción automotriz se incrementó en $115.5 \%$, esto es, más de MXN\$127 mil millones de pesos (mmdp). Sin

Gráfica 1. Valor agregado de la industria automotriz, según periodo censal (miles de millones de pesos base 2010)

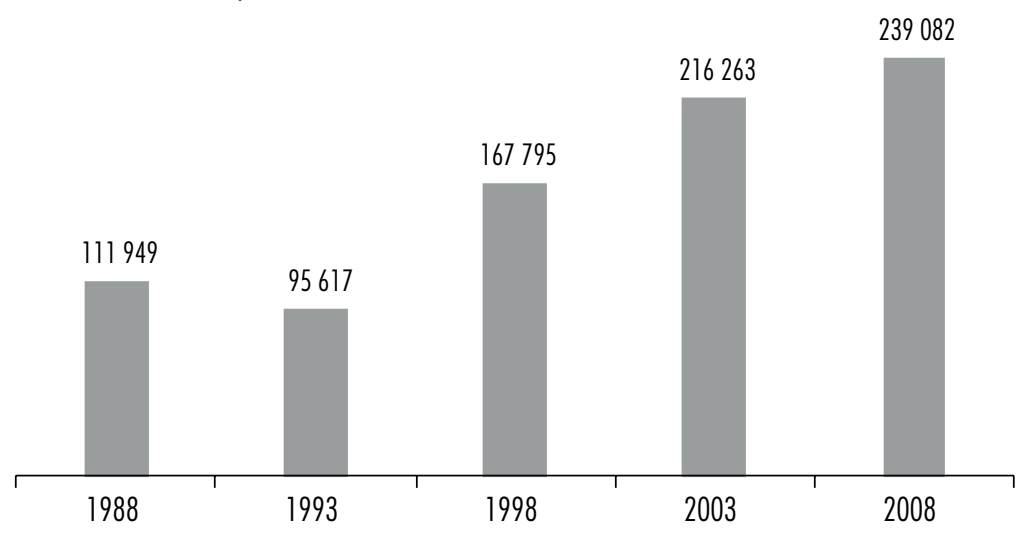

Fuente: elaboración propia. Censos económicos, INEGl. 
embargo, la tendencia no fue siempre en ascenso, pues en 1993 retrocedió $14 \%$ respecto al periodo anterior.

Las variables independientes utilizadas para explicar la producción son: trabajo, capital y consumo de energía eléctrica. El personal ocupado total de las ramas automotrices se expresa en número de trabajadores para cada periodo de tiempo, transformado a logaritmos naturales. El número de trabajadores en esta industria tuvo un comportamiento ascendente en todo el periodo, en particular, al inicio del TLCAN, en 1993-1998, que implicó una expansión de $150.6 \%$, incorporando a 251955 nuevos trabajadores en cinco años como se muestra en la gráfica 2 .

El capital (K) es representado por la variable activos fijos, con valores deflactados con base 2010, y se expresa también como logaritmos naturales. Los activos fijos se incrementaron en $68.2 \%$ en los 20 años correspondientes con el periodo de análisis. Al igual que la variable de Valor Agregado, registró un decremento de 1988 a 1993 de $28.5 \%$, como lo muestra la gráfica 3.

Para controlar por heterogeneidad, se incluyen tres variables en la función de producción. La primera se refiere a los insumos intermedios (energía eléctrica), la segunda es especialización productiva y la tercera población. La introducción de insumos intermedios en la función de producción como una variable de control para estimar el valor del parámetro ha sido probada en otros trabajos como el de Levinsohn y Petri (2003). La propuesta de la variable de control como proxy de insumos intermedios es el consumo de energía eléctrica, deflactada con el INPC base 2010. También, la variable es convertida

Gráfica 2. Personal ocupado total en la industria automotriz, según periodo censal

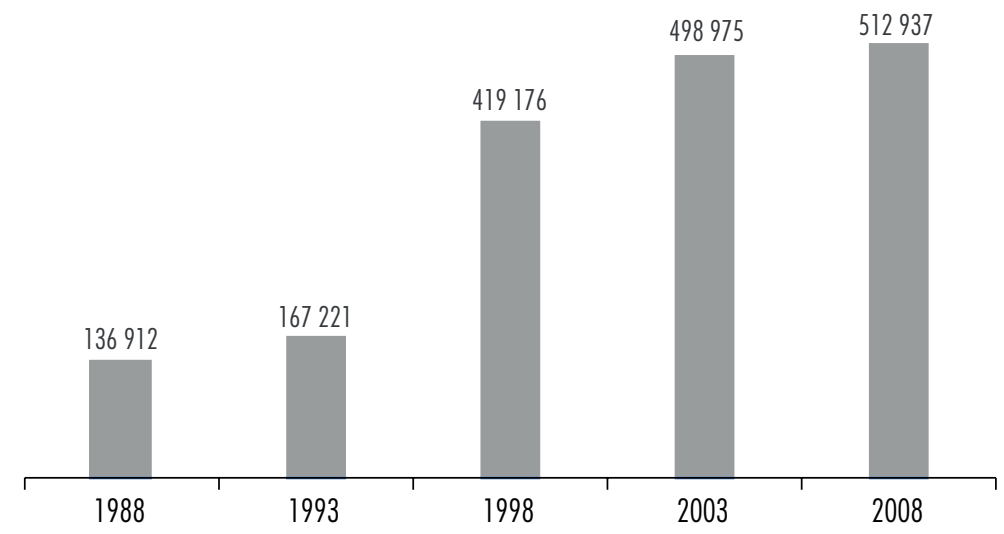

Fuente: elaboración propia. Censos económicos, INEGI. 
Gráfica 3. Activos fijos en la industria automotriz, según periodo censal (miles de millones de pesos base 2010)

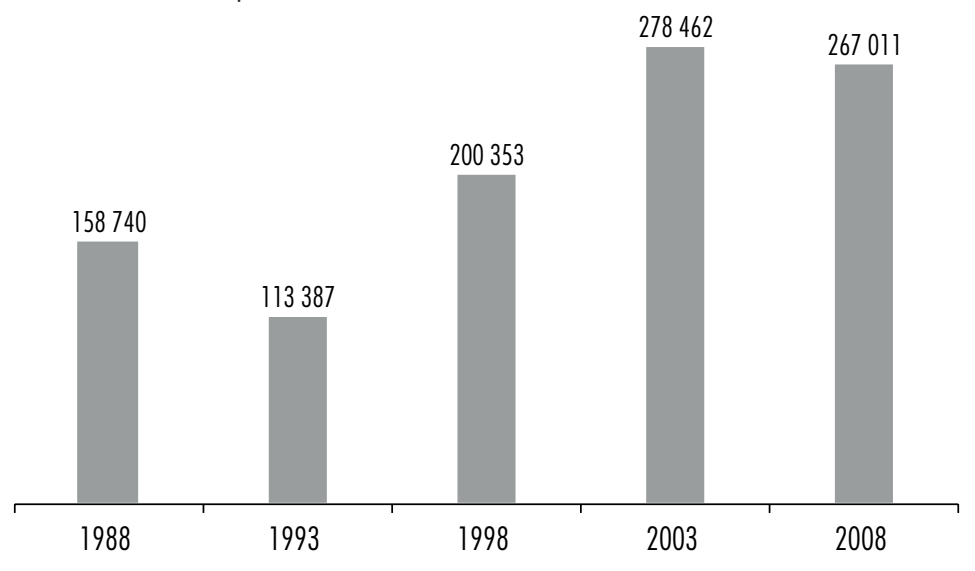

Fuente: elaboración propia. Censos económicos, INEGI.

en logaritmos en la modelación. El consumo de energía eléctrica para 1988 fue estimado por interpolación dada la no disponibilidad de información. La energía eléctrica presenta un comportamiento creciente en todos los periodos. En 1988 la industria automotriz consumió más de MXN\$1 500 mdp, desde ese año hasta 2009 el consumo se incrementó en 498\%.

Para medir el efecto del grado de especialización productiva en la industria automotriz, se incluye un índice de especialización regional automotriz (Asuad, 2001), que se calcula de la siguiente manera:

$$
E s p=\frac{A_{i}}{A t_{i}} / \frac{A_{r}}{A r_{i}}
$$

Donde,

$A_{i}$ : Valor agregado automotriz en el municipio o zona metropolitana i.

$A t_{i}$ : Valor agregado total del municipio o zona metropolitana i.

$A_{r}$ : Valor agregado automotriz nacional.

$A r_{i}$ : Valor agregado total nacional.

El índice permite identificar la especialización económica de las unidades de análisis con sí mismas y con otras áreas geográficas. El análisis del índice 
toma el valor agregado automotriz para comparar la especialización productiva de las regiones de análisis en relación con la especialización nacional. Si $E s p>1$, la región tiene más especialización automotriz que el resto del país; si $E s p<1$, tiene menor especialización automotriz que el resto del país; si $E s p=0$, región y país tienen el mismo grado de especialización automotriz.

Con el fin de controlar la heterogeneidad productiva de las unidades de análisis, se incluye la variable Población dentro de la sección determinística. La fuente de información es el Censo de Población y Vivienda ${ }^{3}$ de los años 1990, 2000 y 2010, para los dos periodos intercensales, 1995 y 2005 del Conteo Intercensal. Específicamente es utilizado el número de habitantes de las unidades geográficas que conforman el análisis. La variable es transformada a logaritmo natural en la sección empírica.

Para modelar la ineficiencia técnica es incluido un grupo de variables con el fin de determinar el impacto que cada una de ellas tiene sobre el aprovechamiento de los factores. Es integrada una variable de escolaridad, definida como el grado promedio de escolaridad de la población de 15 años y más de cada municipio y zona metropolitana -esta última obtenida del promedio de los municipios integrantes; al igual que el dato de población, se obtiene de los censos de población y transformada a logaritmo natural en la sección empírica. Se incluyen variables de tendencia para captar efectos temporales inobservados que impactan en la eficiencia productiva de las unidades de análisis. Con base en la información acerca del año de inicio de operaciones de las plantas automotrices, se construye una variable dicotómica para evaluar el impacto que tienen las armadoras automotrices en las unidades de estudio e identificar si éstas tienen impacto significativo en la reducción de la ineficiencia técnica, dado que las cifras de los censos económicos muestran que existe una cantidad amplia de unidades productivas muy pequeñas y poco sofisticadas.

Para medir efectos regionales de eficiencia técnica, se incluyen cuatro variables instrumentales que capturan si la unidad geográfica de análisis pertenece a una de las cuatro regiones presentadas en el cuadro 1. Las variables instrumentales para la sección empírica, por tanto, son: nor correspondiente a los estados de la región norte del país; cenor con la región centro norte; centro para la región centro y; sur para las entidades de la región del sur. Con la inserción de estas variables es posible contrastar resultados con otras investigaciones sobre el comportamiento de la eficiencia técnica manufacturera regional y verificar si el sector automotriz se asemeja a resultados similares a las manufacturas.

3

<https://www.inegi.org.mx/programas/ccpv/2010/default.html\#Tabulados> 
Eliseo Díaz, Jairo César López y Rafael Garduño

Cuadro 1. Regionalización Banco de México (2011)

\begin{tabular}{llll}
\hline Norte & Centro norte & Centro & Sur \\
\hline Baja California & Aguascalientes & Ciudad de México & Campeche \\
Coahuila de Zaragoza & Baja California Sur & Guanajuato & Chiapas \\
Chihuahua & Colima & Hidalgo & Guerrero \\
Nuevo León & Durango & México & Oaxaca \\
Sonora & Jalisco & Morelos & Quintana Roo \\
Tamaulipas & Michoacán & Puebla & Tabasco \\
& Nayarit & Querétaro & Veracruz \\
& San Luis Potosí & Tlaxcala & Yucatán \\
& Sinaloa & & \\
\hline
\end{tabular}

Fuente: elaboración propia, Banco de México (2011).

Para evaluar el impacto de la apertura comercial del TLCAN en la eficiencia técnica de la manufactura automotriz se incluyó una variable instrumental para su periodo de vigencia, los años posteriores a 1993. De igual forma, para estimar el efecto en la eficiencia productiva relacionado con producir dentro de una zona metropolitana, se incluyó una variable instrumental que distingue si la unidad de análisis es una zona metropolitana o un municipio con actividad automotriz.

\section{ESTIMACIÓN Y DESCRIPCIÓN DE RESULTADOS}

La especialización productiva de la industria automotriz en México se muestra en el cuadro 2, que presenta estados/localidades principales con mayor especialización en la producción automotriz en el país, para los cinco cortes temporales analizados. ${ }^{4}$

Se advierte que las zonas geográficas más especializadas en 1988 contienen municipios del sur del país, como Ocotlán de Morelos en Oaxaca, donde el valor agregado automotriz no es elevado, pero sí representativo. Sin embargo, en periodos posteriores se observa que el norte del país, sobre todo después de la apertura comercial, comienza a especializarse en manufacturas automotri-

4 Los índices de especialización para todas las unidades de análisis son reportados en los Anexos. 
ces. Por ejemplo, dentro de las zonas geográficas presentadas en el cuadro 2, en 1988 y 1993, sólo figuraron dos zonas del norte del país -Saltillo (1988 y 1993) y Chihuahua en (1988). En contraste, para 1998 y años posteriores, la región norte ocupó 4, 5 y 6 espacios, respectivamente, dentro de las principales zonas especializadas, sobre todo en los estados de Coahuila y Chihuahua. Con esta evidencia es posible continuar con la siguiente pregunta de investigación, acerca de cuál es el efecto de la especialización en la eficiencia técnica de la industria automotriz, a la que se dará respuesta más adelante.

Cuadro 2. Localidades con mayores índices de especialización

\begin{tabular}{|c|c|c|c|c|c|c|c|}
\hline \multirow{2}{*}{ Estado } & \multirow[t]{2}{*}{ Localidad } & \multicolumn{5}{|c|}{ Años } & \multirow[t]{2}{*}{ Categoría } \\
\hline & & 1988 & 1993 & 1998 & 2003 & 2008 & \\
\hline Coahuila & Acuña & & & 7.5 & 9.7 & 8.4 & M \\
\hline Sonora & Agua Prieta & & & & & 5.1 & M \\
\hline Aguascalientes & Aguascalientes & & 6.2 & 5.8 & 4.6 & 8.7 & Z.M. \\
\hline Hidalgo & Alfajayucan & & & 10.9 & & 9.3 & M \\
\hline Chihuahua & Chihuahua & 4.3 & & & & & Z. M. \\
\hline Morelos & Cuernavaca & 10.1 & 10.1 & & & & Z. M. \\
\hline Sonora & Hermosillo & & & 6.6 & & & M \\
\hline Chihuahua & Juárez & & & & 4.3 & 5.4 & Z. M. \\
\hline Guanajuato & León & & & 8.9 & 7.2 & & Z. M. \\
\hline Chihuahua & Nvo. Casas Grandes & & & & 18.3 & 6.8 & M \\
\hline Tamaulipas & Nuevo Laredo & & & & & 4.9 & Z. M. \\
\hline Oaxaca & Ocotlán de Morelos & 5.5 & 15.6 & & & & M \\
\hline Querétaro & Pedro Escobedo & 14.9 & 17.8 & 15.6 & & & M \\
\hline Puebla-Tlaxcala & Puebla-Tlaxcala & 3.4 & 7.1 & 7.2 & & & Z. M. \\
\hline Nuevo León & Sabinas Hidalgo & & & 6.1 & 6.5 & & M \\
\hline Coahuila & Saltillo & 12.5 & 8 & 6.7 & 6.6 & 8.7 & Z. $M$. \\
\hline México & Tenango del Valle & & 6.5 & 6.7 & 4.4 & & M \\
\hline Hidalgo & Tepeapulco & 5.5 & 16.9 & & & & M \\
\hline México & Tianguistenco & 9.1 & 17.3 & 8.7 & 8.6 & 13.8 & Z. M. \\
\hline México & Toluca & 4.2 & 6.5 & & & & Z. M. \\
\hline
\end{tabular}

Fuente: elaboración propia a partiir de información de censos económicos, INEGI. 
A continuación, se presentan los resultados de las estimaciones del modelo de eficiencia técnica con el que se pretende encontrar los determinantes a nivel de municipios y zonas metropolitanas; además, con esta estimación se puede observar si el TLCAN contribuyó a reducir la ineficiencia productiva en México y explicar si existe una diferencia significativa en la eficiencia técnica en las regiones de producción automotriz (véase cuadro 3).

Cuadro 3. Resultados del modelo de fronteras estocásticas

Variable dependiente: Iny

\begin{tabular}{|c|c|c|c|c|c|}
\hline Variable & Parámetro & Coef. & Variable & Parámetro & Coef. \\
\hline \multirow[t]{2}{*}{ Intercepto } & $\beta_{0}$ & 0.296 & Ineficiencia & & \\
\hline & & -0.44 & Intercepto & $\delta_{0}$ & 4.41 \\
\hline \multirow[t]{2}{*}{ Capital (K) } & $\beta_{2}$ & 0.232 & & & 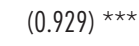 \\
\hline & & 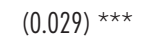 & Especialización & $\delta_{1}$ & -1.327 \\
\hline \multirow[t]{2}{*}{ Trabajo (L) } & $\beta_{3}$ & 0.518 & productiva (esp) & & $(0.325)^{\star \star \star}$ \\
\hline & & 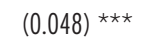 & Escolaridad (esc) & $\delta_{2}$ & -0.819 \\
\hline \multirow[t]{2}{*}{ Energía (E) } & $\beta_{4}$ & 0.12 & & & $(0.496)$ * \\
\hline & & $(0.0427)^{* \star *}$ & Tendencia ( $\mathrm{t}$ ) & $\gamma_{1}$ & -1.278 \\
\hline \multirow{2}{*}{$\begin{array}{l}\text { Especialización } \\
\text { productiva (esp) }\end{array}$} & $\beta_{5}$ & 0.104 & & & $(0.362) * \star \star$ \\
\hline & & $(0.0121)^{\star \star \star}$ & Tendencia cuadrada & $\gamma_{2}$ & 0.183 \\
\hline \multirow[t]{5}{*}{ Población (pob) } & $\beta_{6}$ & 0.3774 & & & $(0.0485)^{\star \star * \star}$ \\
\hline & & 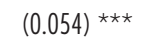 & Armadoras (arm) & $\delta_{3}$ & 0.175 \\
\hline & & & & & $(-0.305)$ \\
\hline & & & Centro norte (cnor) & $\theta_{1}$ & -0.179 \\
\hline & & & & & -0.179 \\
\hline \multirow[t]{2}{*}{ Usigma } & $\sigma_{\mu}$ & 0.64 & Norte (nor) & $\theta_{2}$ & -1.066 \\
\hline & & & & & $(0.368)^{\star \star \star}$ \\
\hline Vsigma & $\sigma_{v}$ & 0.452 & Centro (cen) & $\theta_{3}$ & -0.332 \\
\hline
\end{tabular}




\begin{tabular}{|c|c|c|c|c|c|}
\hline Variable & Parámetro & Coef. & Variable & Parámetro & Coef. \\
\hline Lambda & $\Delta$ & 1.416 & Sur (sur) & $\theta_{4}$ & Colinealidad \\
\hline Observaciones & NxT & 332 & TLCAN (tlcan) & $\delta_{4}$ & $\begin{array}{r}0.832 \\
(0.318) \star \star \star\end{array}$ \\
\hline Log-likelihood & & -313.8 & $\begin{array}{l}\text { Zona Metropolitana } \\
\text { (zm) }\end{array}$ & $\delta_{5}$ & $\begin{array}{r}0.173 \\
-0.15\end{array}$ \\
\hline
\end{tabular}

Fuente: estimaciones propias.

En la estimación de la frontera de posibilidades de producción se encuentra que los coeficientes estimados por máxima verosimilitud, siguiendo la metodología descrita antes (Battese y Coelli, 1995), muestran que en la frontera máxima eficiente los factores productivos Trabajo (L) y Capital (K) son estadísticamente significativos al 99\% y presentan signos positivos, como lo muestra el cuadro 3. El factor trabajo es más elástico (0.51) que el factor capital (0.23) lo que implica mayor participación de la mano de obra en la producción automotriz mexicana. El coeficiente asociado a la energía eléctrica resulta estadísticamente significativo al $99 \%$ y positivo (0.12). La especialización presenta un coeficiente positivo $(0.104)$ y estadísticamente significativo al 99\%, indicando que la especialización productiva contribuye al incremento del valor agregado automotriz. Por último, el coeficiente de población, al igual que los anteriores, es positivo (0.37) y estadísticamente significativo al $99 \%$.

La sección estocástica (o de ineficiencia) del modelo es interpretada de forma distinta, los coeficientes de las variables con signo positivo implican que existe un mayor "alejamiento" de las unidades de análisis en relación con la frontera máxima eficiente, mientras que un signo negativo acerca a las unidades a la frontera óptima, por lo que el resultado esperado es que los coeficientes presenten signo negativo (Cooper et al., 2011).

El coeficiente de especialización productiva de la sección estocástica resulta negativo (-1.32) y es significativo al 99\%, esto confirma la hipótesis planteada de las ventajas de economías de localización y aglomeración. Por su parte, la escolaridad tiene coeficiente negativo $(-0.81)$ y es estadísticamente significativa al 90\%, lo que implica que los años de escolaridad promedio de la población contribuyen a disminuir la ineficiencia técnica automotriz. Trabajos previos (Chávez-Martín del Campo y Fonseca, 2013) muestran coeficientes negativos y significativos para toda la industria manufacturera. 
Los coeficientes de tendencia $(\mathrm{t})$ y tendencia cuadrada $\left(\mathrm{t}^{2}\right)$ son estadísticamente significativos al 99\%, aunque los signos son contrarios. El signo negativo del coeficiente de tendencia lineal $(\mathrm{t})$ implica que existe una disminución de la ineficiencia técnica a lo largo del periodo de análisis. El signo positivo de la tendencia cuadrada $\left(\mathrm{t}^{2}\right)$ implica que la eficiencia técnica es decreciente a lo largo del tiempo, es decir, aunque existe evidencia de que el cambio técnico disminuye la ineficiencia técnica automotriz, ésta tiende a disminuir al paso del tiempo.

Los coeficientes correspondientes con las regiones resultan con disparidades en la significancia estadística. Aunque la región centro-norte presenta un signo esperado, dada la región de comparación no resulta estadísticamente significativa, quizá por el parecido de la estructura productiva con la región de contraste (región sur) que de hecho es omitida por colinealidad. Los coeficientes correspondientes con las regiones norte y centro son estadísticamente significativos al $99 \%$ y presentan signo negativo como se esperaba, esto implica que la ineficiencia técnica automotriz es menor en las regiones norte y centro del país. Otros estudios (Chávez-Martín del Campo y Fonseca, 2013; Álvarez-P. et al., 2017) presentan resultados similares para las manufacturas en estas regiones del país.

Introducido como variable binaria, el TLCAN presenta significancia estadística al 99\% y signo positivo (0.83). La ineficiencia técnica en el sector manufacturero aumenta después del TLCAN, lo cual coincide con hallazgos de otros autores (Álvarez-P. et al., 2017), quienes advierten que los resultados pueden tener efectos mezclados con otros acontecimientos. El coeficiente asociado a la etapa de liberalización debe interpretarse con precaución, ya que el efecto del TLCAN probablemente se mezcle con otros eventos importantes como la crisis del "efecto tequila" (1995), los acontecimientos terroristas del 11 de septiembre en 2001, o el ingreso de China a la omc.

Producir en una zona metropolitana no resulta ser significativo, como lo indica el coeficiente asociado con esta variable binaria. Por último, el valor de $\lambda$ es resultado del cociente de las desviaciones estándar de $u$ y $v\left(\sigma_{u} / \sigma_{v}\right)$ e indica la ineficiencia del modelo que no es explicada por las variables independientes. En el cuadro 4 se muestran las unidades de análisis (zonas metropolitanas y municipios) que presentaron actividad automotriz de acuerdo con el rango de eficiencia al que pertenecen, identificando además el tipo de unidad territorial, la entidad federativa y la región en la que está localizada esa entidad. Los resultados fueron divididos en cuatro partes y el cuadro sólo presenta las unidades territoriales que resultan con mayor eficiencia productiva y el total de resultados se presenta en los Anexos estadísticos. 


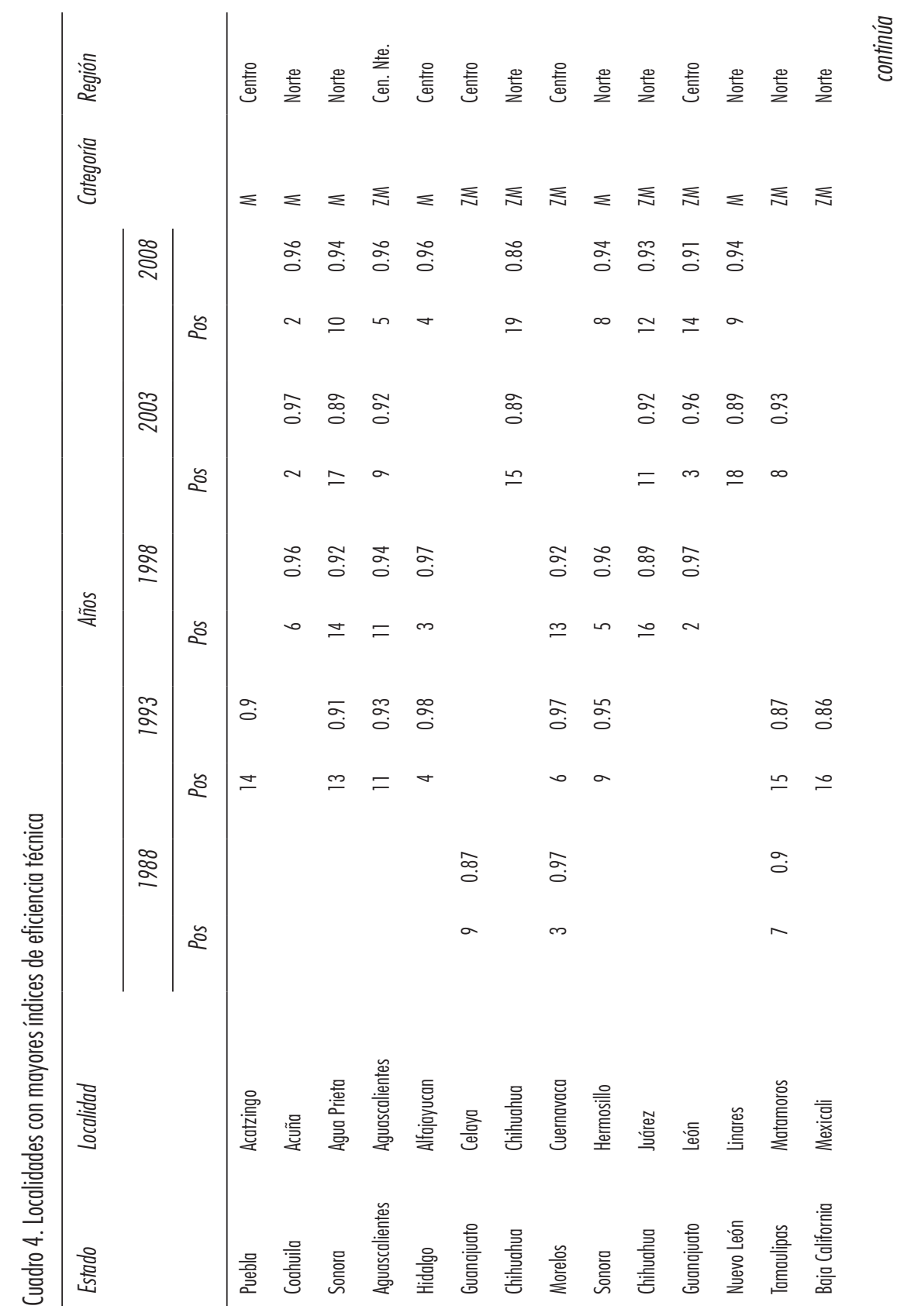




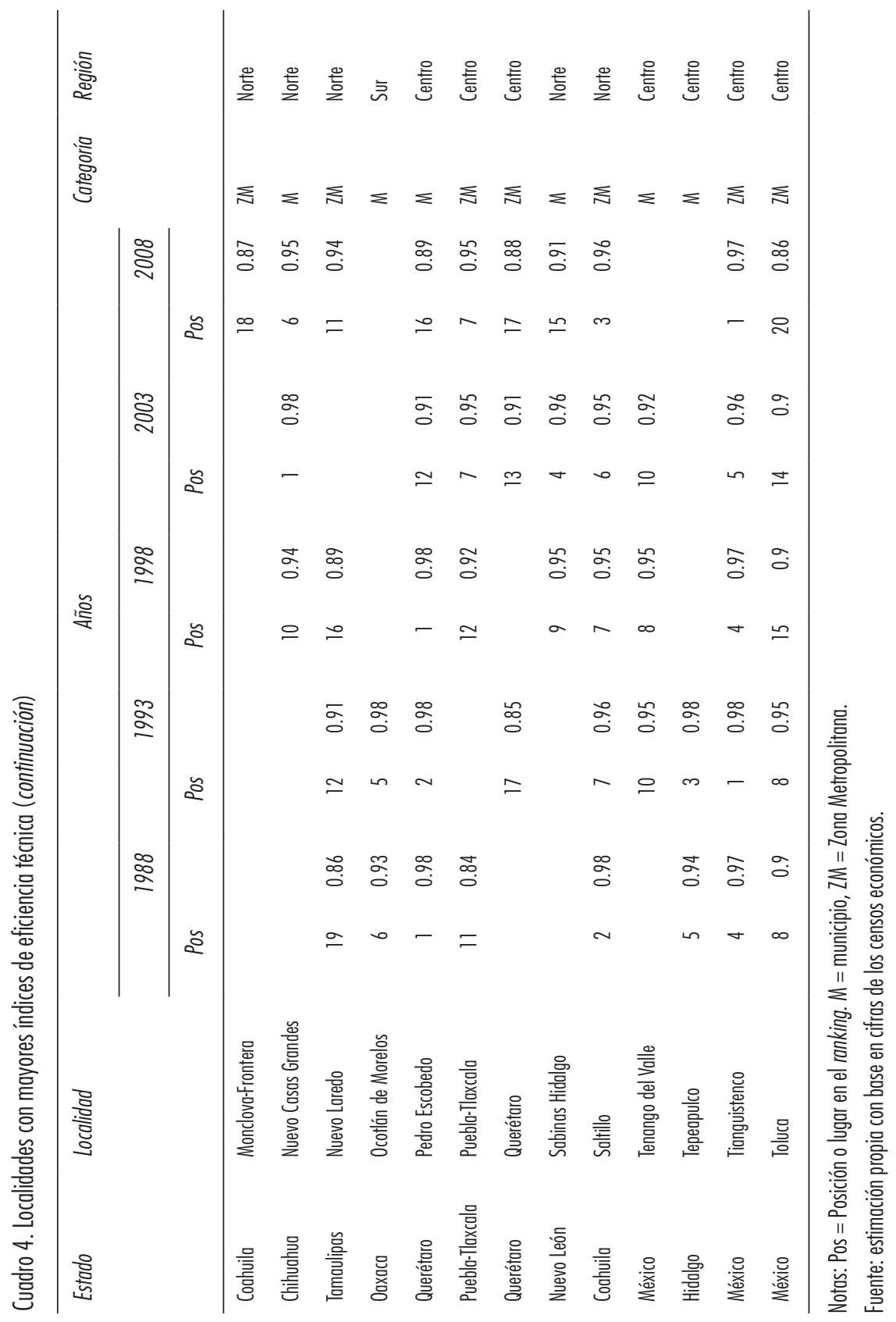


La eficiencia se obtiene a partir del modelo estimado previamente y se evalúa cada una de las unidades de análisis de acuerdo con los valores de las variables en cada periodo. Las zonas más eficientes corresponden a dos regiones de la cuatro consideradas, que son las regiones norte y centro del país. Destaca el caso de Aguascalientes que quedó agrupada en la región centro-norte y uno de la región sur, Ocotlán de Morelos en Oaxaca que presentó un elevado nivel de eficiencia entre 1988-1993, es decir, previo al inicio del TLCAN. Por lo demás, de los 28 municipios o zonas metropolitanas en el grupo de los más eficientes, 13 corresponden a la región norte, 12 a la región centro y uno a la región centro-norte.

La apertura económica no sólo se expresó en una concentración de la eficiencia productiva del sector automotriz, del centro o sur hacia el norte del país, sino también se registran reacomodos de la capacidad productiva en el sector a nivel de los propios estados. Es el caso de Guanajuato, en donde antes del tLCAN la zona metropolitana de Celaya presentó alta eficiencia (19881993), pero después es la zona metropolitana de León la que cobra mayor nivel de eficiencia (1998-2008) en la que llega a ocupar la segunda y tercera posición en el ranking de eficiencia productiva en 1998 y 2003.

La industria automotriz se origina en la etapa de sustitución de importaciones, la apertura económica a mediados de los ańos noventa reestructura el sector y atrae nuevos establecimientos que coexisten con la parte de la industria de más arraigo en México. La parte de la industria del primer periodo tiene alta eficiencia en la fase anterior al TLCAN, pero después tiende a ser referente en este sentido. Son los casos de la ZM en Cuernavaca, en parte Mexicali, entre otros. Otro grupo son los que vienen desde el pasado con una industria automotriz fuerte, y que se fortalecen aún más en la fase posterior del TLCAN son los casos de Saltillo, Coahuila, Tianguistengo y Toluca, en el Estado de México, Escobedo en Querétaro, Nuevo Laredo en Tamaulipas, la zM PueblaTlaxcala. Los nuevos desarrollos industriales automotrices asociados a la apertura económica son los de León, Guanajuato, Querétaro capital, en Sonora, Hermosillo y Agua Prieta, y en Coahuila, Acuña.

Otro caso es el estado de Chihuahua donde las zM de Ciudad Juárez y Chihuahua ilustran el caos de dos regiones dentro de un mismo estado que disputan preeminencia en la eficiencia productiva que, partiendo de un nivel similar, Juárez termina siendo más eficiente. Ambos procesos ocurren en la era posterior al TLCAN, de manera que no sólo debe compararse la situación del antes y después de la apertura económica, sino también se puede evaluar los saldos de la propia apertura dentro de regiones beneficiadas por el libre comercio. 
Los resultados obtenidos y descritos líneas arriba sugieren que la productividad en el sector automotriz se explica en mayor proporción por la acción de una fuerza laboral adiestrada y disciplinada, que por la contribución de las inversiones de capital hechas en el sector. La puesta en vigor del TLCAN parece no haber incidido en hacer más eficiente a las empresas del sector, al menos en forma estocástica y para el conjunto de la muestra, al contrario, el coeficiente asociado a la liberalización comercial es positivo. Debe aclararse que no fue posible aislar los efectos del TLCAN de otros eventos económicos e históricos que impactaron en la economía mexicana al inicio del TLCAN, como el ingreso de China a la OMc o la llamada crisis del llamado "efecto tequila" de mediados de los años noventa.

Los resultados muestran además que las regiones con mayor eficiencia son la región centro y norte del país, y que concuerdan con las zonas de mayor presencia automotriz del país, como los estados de Guanajuato, Coahuila, Sonora, Puebla o Estado de México.

El capital humano medido por la escolaridad es una variable significativa que impacta en el nivel de eficiencia técnica automotriz. Por lo que el nivel educativo de los trabajadores contribuye a la mejora del aprovechamiento de los recursos productivos. El norte del país, particularmente después de la apertura comercial, comienza a especializarse en manufacturas automotrices, mostrando que las principales zonas geográficas se localizan en Coahuila, Chihuahua, Tamaulipas y Sonora. En relación con el impacto de la especialización en la eficiencia técnica, se obtuvo un coeficiente significativo al $99 \%$ y negativo, con esto se comprueba la hipótesis de que la especialización productiva contribuye a mejorar los niveles de eficiencia técnica dadas las ventajas que ofrece: ventajas de información, disminución de costos, cercanía a la competencia y a clientes, como postulara el enfoque teórico adoptado en este trabajo (Krugman, 1991).

El coeficiente asociado con la variable binaria para indicar si la unidad de análisis pertenece a una zona metropolitana no resultó estadísticamente significativo, por lo que no se encontró evidencia de efectos inobservados de economías de urbanización (Goldstein y Gronberg, 1984).

La eficiencia técnica del norte del país, en promedio, fue mayor que la eficiencia de otras regiones desde el inicio del periodo de análisis como se muestra en la gráfica 4. La región sur tuvo un comportamiento opuesto al igual que la región centro, aunque la primera con una disminución mucho mayor, específicamente de $41.2 \%$ en términos porcentuales. La eficiencia promedio nacional, se incrementó en $5.8 \%$ en todo el periodo, aunque desde su nivel más elevado en 1993 ha ido decreciendo. 
Grática 4. Índice de eficiencia promedio regional, 1988 - 2008

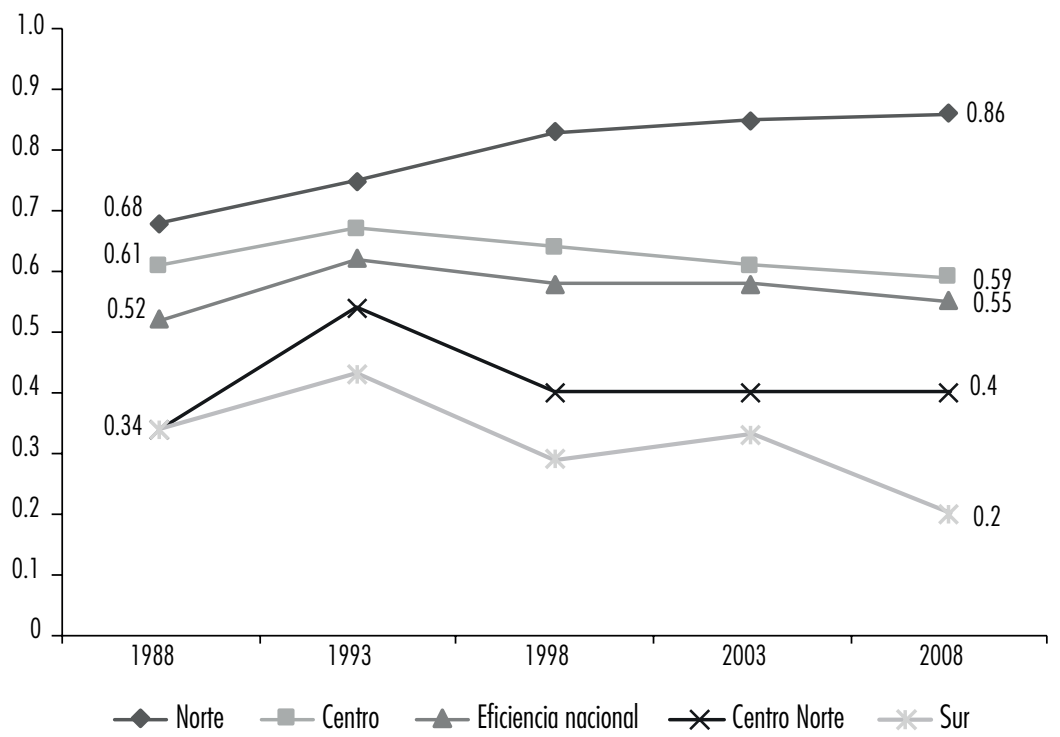

Fuente: estimaciones propias, Battese y Coelli (1995).

\section{CONCLUSIONES}

Las teorías y técnicas de estimación revisadas y aplicadas a los datos censales disponibles para el sector automotriz de México permiten hacer una verificación positiva de la hipótesis de investigación según la cual, el desarrollo reciente de la industria automotriz impulsó la especialización productiva de regiones en la exportación de bienes automotrices y estas regiones están en la frontera de posibilidades de producción de esta industria. Así como de las preguntas de investigación específicas propuestas como guía de la presente investigación.

Los resultados obtenidos y descritos en el apartado anterior sugieren que la productividad en el sector automotriz se explica en mayor proporción por la acción de una fuerza laboral adiestrada y disciplinada, más que por la contribución de las inversiones de capital hechas en el sector. Sin embargo, ambas contribuyen a la dinámica de la productividad, tal como se observa en los resultados obtenidos. Este efecto pareciera contradecir tanto la evidencia empírica como el postulado que asocia la innovación técnica a las inversiones de capital, sin embargo, la base de datos utilizada -cifras censales nacionales no sólo de las regiones automotrices propiamente-, no permiten captar estos impactos esperados. 
En la frontera máxima eficiente, los factores productivos son estadísticamente significativos al $99 \%$ y mostraron signo positivo. El factor trabajo presenta mayor elasticidad que el factor capital lo que implica mayor participación de la mano de obra en la frontera eficiente de la producción automotriz. Asimismo, relacionado al uso de bienes intermedios, el coeficiente asociado a la energía eléctrica resulta estadísticamente significativo y positivo. Por su parte, como un resultado esperado, la especialización es un determinante robusto de la frontera de posibilidades de producción, tanto como la variable población.

Cabe destacar que la mayor parte de las variables de control agregadas en el estudio -con el fin de dar respuesta a las preguntas que guiaron la investigación-, muestran tendencias que explican la distancia de las regiones a la frontera eficiente de producción.

Los resultados confirman que la especialización productiva aproxima a las regiones a la frontera eficiente de producción, también lo hace la escolaridad que contribuye a disminuir la ineficiencia técnica automotriz, tal como coinciden trabajos previos como el de Chávez-Martín del Campo y Fonseca (2013). La influencia de la tendencia en la eficiencia técnica mostró signos contrastantes. En el periodo de análisis, la tendencia de corto plazo tendió a reducir la distancia respecto a la frontera eficiente, pero a largo plazo esa influencia se revirtió.

Regionalmente, se muestra que la ineficiencia técnica automotriz es menor en las regiones norte y centro del país, que son las que concentrar las principales actividades industriales en el sector, lo cual coincide también con resultados del índice de especialización productiva. Otros trabajos han obtenido resultados similares para la industria manufactura en estas regiones del país (Chávez-Martín del Campo y Fonseca, 2013 y Álvarez-P. et al., 2017).

La excepción es la puesta en vigor del TLCAN, que parece no haber incidido en hacer más eficiente a las empresas del sector, pues muestra un impacto negativo en la eficiencia productiva automotriz. Muchos factores de origen interno y externo pueden ayudar a explicar este resultado como la escala de la muestra analizada, el periodo o la regionalización para la actividad automotriz. En cualquier sentido, una investigación más acotada puede contribuir a esclarecer en mejor término la influencia de la integración comercial impulsada. 


\section{BIBLIOGRAFÍA}

Acevedo, M. C. y Ramírez, J. (2005), "Diferencias regionales en la eficiencia técnica del sector confecciones en Colombia: un análisis de fronteras estocásticas", INNOVAR. Revista de Ciencias Administrativas y Sociales, vol. 15, núm. 26, Colombia, Universidad Nacional de Colombia.

Aigner, D., Lovell, C. A. K. y Schmidt, P. (1977), "Formulation and estimation of stochastic frontier production function models", Journal of Econometrics, vol. 6, núm. 1, DOI <https://doi.org/10.1016/0304-4076(77)900 $52-5>$

Álvarez-P., A. (coord.) (2013), La medición de la eficiencia y la productividad, Madrid, Ediciones Pirámide, colección Economía y Empresa.

Álvarez-P., A., Garduño, R. y Núñez, H. M. (2015), "Estimating the technical efficiency of mexican states", Documentos de Trabajo del CIDE, Versión: 588, In Progress, DoI <https://doi.org/10.13140/RG.2.1.5089.1680>

(2017), "Mexico's north-south divide: the regional distribution of state inefficiency 1988-2008”, Papers in Regional Science, vol. 96, núm. 4, DOI <https://doi.org/10.1111/pirs.12303>

Arellano, P. L. y Cortés, J. H. (2010), "Análisis de la eficiencia técnica relativa de la agroindustria azucarera: el caso de México", Revista Mexicana de Agronegocios, vol. 26, enero-junio, México, Sociedad Mexicana de Administración Agropecuaria A.C.

Arrow, K. J. (1962), "The economic implications of learning by doing", The Review of Economic Studies, vol. 29, núm. 3, DoI <https://doi.org/10. 1007/978-1-349-15430-2_11>

Arteaga, A. (2003), Integración productiva y relaciones laborales en la industria automotriz en México, México, Universidad Autónoma Metropolitana y Plaza y Valdés Editores.

Asociación Mexicana de la Industria Automotriz (AMIA) (2018), Diálogo con la industria automotriz 2018-2024, México, AMIA.

Asuad, N. (2001), Economía regional y urbana, México, Benemérita Universidad Autónoma de Puebla, Colegio de Puebla, aefe-unam.

Audretsch, D. y Feldman, M. (1996), "R\&D spillovers and the geography of innovation and production", American Economic Review, vol. 86, núm. 3. Recuperado de <http://www.jstor.org/stable/2118216>

Banco de México (2011), "Reporte sobre las economías regionales", octubrediciembre 2011". Recuperado de <http://www.banxico.org.mx/publica ciones-y-prensa/reportes-sobre-las-economias-regionales/\%7B08C41905A999-3CA8-6353-2B8547674E5E\%7D.pdf> 
Bannister, G. J. y Stolp, C. (1995), "Regional concentration and efficiency in mexican manufacturing", European Journal of Operational Research, vol. 80, núm. 3, DOI <https://doi.org/10.1016/0377-2217(94)00144-2>

Battese, G. E. y Coelli, T. (1995), "A model for technical inefficiency effects in a stochastic frontier production function", Empirical Economics, vol. 20, núm. 2, DOI <https://doi.org/10.1007/BF01205442>

Becerril, O., Álvarez, I. y Nava, R. (2012), "Frontera tecnológica y eficiencia técnica de la educación superior en México", Revista Mexicana de Investigación Educativa, vol. 17, núm. 54, México, Consejo Mexicano de Investigación Educativa, A.C.

Chávez-Martín del Campo, J. C. y Fonseca, F. J. (2013), “Technical efficiency, technological development, and the labor productivity gap in mexican manufacturing", Regional and Sectoral Economic Studies, vol. 13, núm. 2, España, Guest.

Christensen, L. R., Jorgenson, D. W. y Lau, L. J. (1973), "Transcendental logarithmic production frontier", The Review of Economics and Statistics, vol. 55, núm.1, DOI <https://doi.org/10.2307/1927992>

Cobb, C. W. y Douglas, P. H. (1928), "A theory of production", The American Economic Review, vol. 18, núm. 1, DOI <https:/doi.org/10.1515/ humr.1998.11.2.161>

Consejo Nacional de Población (conapo) (2010), Delimitación de las zonas metropolitadas de México 2010, México, CONAPo.

Cooper, W. W., Seiford, L. M. y Zhu, J. (2011), "Data envelopment analysis: history, models and interpretations", Handbook on Data Envelopment Analysis, DOI <https://doi.org/10.1007/978-1-4419-6151-8_1>

Covarrubias, A. (2014), Explosión de la industria automotriz en México: de sus encadenamientos actuales a su potencial transformador, México, Fundación Friedrich-Ebert-Stiftung. Recuperado de <http://library.fes.de/pdf-files/ bueros/mexiko/10645.pdf>

Cullinane, K., Wang, T.-F., Song, D.-W. y Ji, P. (2006), “The technical efficiency of container ports: comparing data envelopment analysis and stochastic frontier analysis", Transportation Research Part A: Policy and Practice, vol. 40, núm. 4, DOI <https://doi.org/10.1016/j.tra.2005.07.003>

Dombois, R. (1985), La producción automotriz y el mercado de trabajo en un pais en desarrollo. Un estudio sobre la industria automotriz mexicana. IIVG/ dp85-206, Berlín, International Institute for Comparative Social Research and Labor Policy, julio.

Driffield, N. y Munday, M. (2001), "Foreign manufacturing, regional agglomeration and technical efficiency in UK industries: a stochastic produc- 
tion frontier approach", Regional Studies, vol. 35, núm. 5, DoI <https:// doi.org/10.1080/713693833>

Eberts, R. W. y McMillen, D. P. (1999), "Agglomeration economies and urban public infrastructure”, en P. Cheshire y E. S. Mills (eds.), Handbook of Regional and Urban Economics, vol. 3, Applied Urban Economics, Amsterdam, Elsevier Science, DOI <https://doi.org/10.1016/S1574-0080 (99) $80007-8>$

Farrell, M. (1957), "The measurement of productive efficiency", Journal of the Royal Statistical Society, Series A (General) vol. 120, núm. 3, DOI <https:// doi.org/10.2307/2343100>

Feldman, M. P. (1994), "The university and economic development: the case of Johns Hopkins University and Baltimore", Economic Development Quarterly, vol. 8, núm. 1, febrero, DOI <https://doi.org/10.1177/ 089124249400800105>

Fujita, M., Krugman, P. y Venables, A. (1999), The spatial economy: cities, regions and international trade, Cambridge, MA, MIT Press.

Goldstein, G. S. y Gronberg, T. J. (1984), "Economies of scope and economies of agglomeration", Journal of Urban Economics, vol. 16, núm. 1, DOI <https://doi.org/10.1016/0094-1190(84)90052-4>

Gutiérrez, G. A. (2011), "Eficiencia industrial en las regiones de México", EconoQuantum, vol. 7, núm. 2, <http://www.scielo.org.mx/scielo.php? script $=$ sci_arttext\&pid=S1870-66222011000100006>

Instituto Nacional de Estadística y Geografía (INEGI) (2018), Conociendo la industria automotriz (noviembre), México, INEGI.

Jacobs, J. (1969), "Strategies for helping cities", American Economic Review, vol. 59, núm. 4, Nashville, TN, American Economic Association.

Jaffe, A. B. (1989), "Real effects of academic research", American Economic Review, American Economic Association, vol. 79, núm. 5, diciembre, Nashville, TN, American Economic Association.

Kirkley, J. E., Squires, D. y Strand, I. E. (1995), "Assessing technical efficiency in commercial fisheries: the mid-atlantic sea efficiency in scallop fishery", American Journal of Agricultural Economics, vol. 77, núm. 3, agosto, DOI <https://doi.org/10.2307/1243235>

Koopmans, T. C. (1951), "Efficient allocation of resources", Econometrica, vol. 19, núm. 4, DOI <https://doi.org/10.2307/1907467>

Krugman, P. (1980), "Scale economies, product differentiation, and the pattern of trade", The American Economic Review, vol. 70, núm. 5, Nashville, TN, American Economic Association. 
(1981), "Intraindustry specialization and the gains from trade", Journal of Political Economy, vol. 89, núm. 5, University of Chicago Press, octubre.

(1991), Geography and trade, Cambridge, MA, MIT Press.

Krugman, P. y Venables, A. (1995), "Globalization and the inequality of nations”, The Quarterly Journal of Economics, vol. 110, núm. 4, DOI <10.3386/ w5098>

Levinsohn, J. y Petrin, A. (2003), "Estimating production functions using inputs to control for unobservables", The Review of Economic Studies, vol. 70, núm. 2, 317-341, DOI <https://doi.org/10.1111/1467-937X.00246>

Marshall, A. (1920), "Industry and trade", History of Economic Thought Books, McMaster University Archive for the History of Economic Thought, 3a. edición, Canadá. Recuperado de <https://socialsciences.mcmaster.ca/ econ/ugcm $/ 3113 / \mathrm{marshall} /$ Industry\%26Trade.pdf>

Mendoza, J. E. (2003), "Especialización manufacturera y aglomeración urbana en las grandes ciudades de México", Economía, Sociedad y Territorio, vol. 4, núm. 13, DOI <http://dx.doi.org/10.22136/est002003329>

Miranda, A. V. (2007), "La industria automotriz en México", Revista de Contaduría y Administración, núm. 221, México, unAm.

Montiel, S.H. T. (2012), "El impacto de la apertura comercial en la eficiencia técnica de las manufacturas en México: un análisis por entidad federativa", Revista de Economía, vol. XXIX, núm. 79, México, Universidad Autónoma de Yucatán, julio-diciembre.

Navarro, J. C. y Torres, Z. (2006), "Eficiencia técnica y asignativa del sector eléctrico en México en su fase de distribución: un análisis a través de los modelos de frontera DEA", Mundo Siglo XXI, núm. 7, México, CIECAS-IPN. Peón, S. y Casimiro, I. (2017), "Recaudación potencial, eficiencia recaudatoria y transferencias federales: un análisis para las entidades federativas en México utilizando el modelo de frontera", EconoQuantum, DOI <http:// dx.doi.org/10.18381/eq.v14i1.6541>

Pitt, M. M. y Lee, L. F. (1981), "The measurement and sources of technical inefficiency in the Indonesian weaving industry", Journal of Development Economics, vol. 9, núm. 1, DOI <https://doi.org/10.1016/03 04-3878(81)90004-3>

Porter, M. (1982), Competitive strategy. Technics for analyzing industries and competitors, The Free Press, a division of Macmillan Publishing Co., New York, Free Press.

Reinhard, S., Knox Lovell, C. A. y Thussen, G. (1999), "Econometric estimation of technical and environmental efficiency: an application to dutch 
dairy farms", American Journal of Agricultural Economics, 81, febrero, DOI <https://doi.org/10.2307/1244449>

Romer, P. M. (1986), "Increasing returns and long run growth", Journal of Political Economy, vol. 94, núm.5, octubre, EUA, University of Chicago Press.

Seyoum, E. T., Battese, G. E. y Fleming, E. M. (1998), “Technical efficiency and productivity of maize producers in eastern Ethiopia: a study of farmers within and outside the Sasakawa-Global 2000 project", Agricultural Economics, vol. 19, núm. 3, DOI <https://doi.org/10.1016/S01695150(98)00037-1>

Tovar, B. y Martín-Cejas, R. R. (2010), “Technical efficiency and productivity changes in spanish airports: a parametric distance functions approach", Transportation Research Part E: Logistics and Transportation Review, vol. 46, núm. 2, DOI <https://doi.org/10.1016/j.tre.2009.08.007>

Unger, K. y Chico, R. (2004), "La industria automotriz en tres regiones de México. Un análisis de clusters", El Trimestre Económico LXXI (284), México, Fondo de Cultura Económica.

Venables, A. (1996), "Equilibrium locations of vertically linked industries", International Economic Review 37, DOI <10.2307/2527327>

\section{ANEXOS}

Cuadro A1. Estadística descriptiva de las variables del modelo

\begin{tabular}{lrrrrr}
\hline Año & 1988 & 1993 & 1998 & 2003 & 2008 \\
\hline Variable: Trabajo: (L) & & & & & \\
Observaciones & 72 & 72 & 72 & 72 & 72 \\
$\begin{array}{l}\text { Promedio } \\
\text { Desv. Est. }\end{array}$ & 1868 & 2279 & 5296 & 6028 & 6275 \\
Mínimo & 5478 & 6219 & 13390 & 12535 & 12332 \\
Máximo & 2 & 2 & 2 & 1 & 2 \\
Variable: Capital (K) & 41814 & 45903 & 96894 & 81915 & 76485 \\
Observaciones & 72 & & & & 72 \\
Promedio & 2174856 & 1547622 & 2718904 & 3817511 & 3621275 \\
Desv. Est. & 6865632 & 4649464 & 6737338 & 12000000 & 8863489
\end{tabular}

continúa 


\section{Eliseo Díaz, Jairo César López y Rafael Garduño}

Cuadro A1. Estadística descriptiva de las variables del modelo (continuación)

\begin{tabular}{|c|c|c|c|c|c|}
\hline Año & 1988 & 1993 & 1998 & 2003 & 2008 \\
\hline Mínimo & 13 & 0 & 50 & 0 & 11 \\
\hline Máximo & 41700000 & 31400000 & 31700000 & 86600000 & 51200000 \\
\hline \multicolumn{6}{|c|}{ Variable: Consumo de energía eléctrica (E) } \\
\hline Observaciones & 72 & 72 & 72 & 72 & 72 \\
\hline Promedio & 18616 & 28383 & 45409 & 68029 & 119249 \\
\hline Desv. Est. & 49207 & 66045 & 92774 & 139708 & 226228 \\
\hline Mínimo & 0 & 3 & 2 & 1 & 3 \\
\hline Máximo & 285376 & 333431 & 389999 & 677434 & 913542 \\
\hline \multicolumn{6}{|c|}{ Variable: Valor Agregado (Y) } \\
\hline Observaciones & 72 & 72 & 72 & 72 & 72 \\
\hline Promedio & 1536475 & 1317774 & 2264689 & 2794772 & 3151164 \\
\hline Desv. Est. & 5024446 & 3726894 & 5059971 & 6440608 & 6715709 \\
\hline Mínimo & 0 & 88 & 41 & 73 & 3 \\
\hline Máximo & 31400000 & 25200000 & 28300000 & 38500000 & 35500000 \\
\hline
\end{tabular}

Fuente: elaboración propia con base en datos de los censos económicos de los años incluidos.

Cuadro A2. Índices de especialización productiva de las unidades de análisis

\begin{tabular}{|c|c|c|c|c|c|c|}
\hline \multirow[t]{2}{*}{ Clave } & \multirow{2}{*}{$\begin{array}{l}\text { Zona Metropolitana / } \\
\text { municipio }\end{array}$} & \multicolumn{5}{|c|}{ Eficiencia } \\
\hline & & 1988 & 1993 & 1998 & 2003 & 2008 \\
\hline 1 & Aguascalientes & 2.000 & 6.200 & 5.800 & 4.600 & 8.700 \\
\hline 2 & Tijuana & 0.100 & 0.200 & 0.200 & 0.200 & 0.700 \\
\hline 3 & Mexicali & 0.600 & 1.400 & 1.300 & 1.100 & 1.400 \\
\hline 4 & La Laguna & 0.400 & 1.100 & 0.500 & 0.700 & 1.100 \\
\hline 5 & Saltillo & 12.500 & 8.000 & 6.700 & 6.600 & 8.700 \\
\hline 6 & Monclova-Frontera & 0.000 & 0.100 & 0.700 & 2.100 & 2.100 \\
\hline 8 & Colima-Villa de Álvarez & 0.000 & 0.000 & 0.000 & 0.100 & 0.200 \\
\hline 10 & Tuxtla Gutiérrez & 0.000 & 0.000 & 0.000 & 0.300 & 1.100 \\
\hline 11 & Juárez & 0.300 & 0.500 & 3.700 & 4.300 & 5.400 \\
\hline 12 & Chihuahua & 4.300 & 0.400 & 2.800 & 3.400 & 2.700 \\
\hline 13 & Valle de México & 0.600 & 0.800 & 0.100 & 0.100 & 0.100 \\
\hline
\end{tabular}




\begin{tabular}{|c|c|c|c|c|c|c|}
\hline \multirow[t]{2}{*}{ Clave } & \multirow{2}{*}{$\begin{array}{l}\text { Zona Metropolitana / } \\
\text { municipio }\end{array}$} & \multicolumn{5}{|r|}{ Eficiencia } \\
\hline & & 1988 & 1993 & 1998 & 2003 & 2008 \\
\hline 14 & León & 0.100 & 0.100 & 8.900 & 7.200 & 4.300 \\
\hline 18 & Pachuca & 0.400 & 0.600 & 0.000 & 0.000 & 0.000 \\
\hline 19 & Tulancingo & 0.100 & 0.100 & 0.100 & 0.000 & 0.000 \\
\hline 21 & Guadalajara & 0.100 & 0.600 & 0.200 & 0.300 & 0.400 \\
\hline 24 & Toluca & 4.200 & 6.500 & 3.500 & 3.300 & 2.600 \\
\hline 25 & Morelia & 0.000 & 0.000 & 0.000 & 0.000 & 0.000 \\
\hline 26 & Zamora-Jacona & 0.000 & 0.000 & 0.000 & 0.000 & 0.000 \\
\hline 28 & Cuernavaca & 10.100 & 10.100 & 3.600 & 1.500 & 1.900 \\
\hline 29 & Cuautla & 0.000 & 0.100 & 1.300 & 2.000 & 2.300 \\
\hline 30 & Tepic & 0.000 & 0.000 & 0.000 & 0.000 & 0.000 \\
\hline 31 & Monterrey & 0.400 & 0.600 & 0.900 & 1.000 & 1.100 \\
\hline 32 & Oaxaca & 0.000 & 0.100 & 0.000 & 0.000 & 0.000 \\
\hline 34 & Puebla-Tlaxcala & 3.400 & 2.800 & 5.200 & 7.100 & 7.200 \\
\hline 35 & Tehuacán & 0.000 & 0.000 & 0.000 & 0.000 & 0.000 \\
\hline 36 & Querétaro & 2.100 & 2.200 & 2.500 & 3.600 & 3.400 \\
\hline 38 & SLP-S. de Graciano Sánchez & 0.800 & 0.800 & 1.100 & 1.700 & 1.800 \\
\hline 43 & Reynosa-Río Bravo & 0.000 & 0.100 & 1.500 & 0.900 & 0.800 \\
\hline 44 & Matamoros & 2.800 & 1.900 & 2.000 & 3.600 & 4.600 \\
\hline 45 & Nuevo Laredo & 1.900 & 3.400 & 2.700 & 2.600 & 4.900 \\
\hline 46 & Tlaxcala-Apizaco & 0.000 & 0.300 & 0.400 & 0.100 & 0.200 \\
\hline 47 & Veracruz & 0.000 & 0.000 & 0.000 & 0.000 & 0.000 \\
\hline 49 & Poza Rica & 0.000 & 0.000 & 0.000 & 0.000 & 0.000 \\
\hline 50 & Orizaba & 0.000 & 0.000 & 0.000 & 0.000 & 0.000 \\
\hline 53 & Córdoba & 0.000 & 0.000 & 0.000 & 0.000 & 0.000 \\
\hline 54 & Acayucan & 0.000 & 0.000 & 0.000 & 0.000 & 0.000 \\
\hline 57 & Celaya & 2.000 & 1.500 & 2.700 & 1.600 & 2.100 \\
\hline 58 & Tianguistenco & 9.100 & 17.300 & 8.700 & 8.600 & 13.800 \\
\hline 2001 & Ensenada & 0.100 & 0.100 & 0.400 & 0.600 & 0.500 \\
\hline 5002 & Acuña & 0.400 & 0.800 & 7.500 & 9.700 & 8.400 \\
\hline 7019 & Comitán de Domínguez & 0.000 & 0.100 & 0.100 & 0.100 & 0.000 \\
\hline 8050 & Nuevo Casas Grandes & 0.000 & 0.100 & 4.900 & 18.300 & $\begin{array}{r}6.800 \\
\text { continúa }\end{array}$ \\
\hline
\end{tabular}


Eliseo Díaz, Jairo César López y Rafael Garduño

Cuadro A2. Índices de especialización productiva de las unidades de análisis (continuación)

\begin{tabular}{|c|c|c|c|c|c|c|}
\hline \multirow[t]{2}{*}{ Clave } & \multirow{2}{*}{$\begin{array}{l}\text { Zona Metropolitana / } \\
\text { municipio }\end{array}$} & \multicolumn{5}{|c|}{ Eficiencia } \\
\hline & & 1988 & 1993 & 1998 & 2003 & 2008 \\
\hline 10005 & Durango & 0.000 & 0.000 & 0.000 & 0.000 & 0.400 \\
\hline 11017 & Irapuato & 0.100 & 0.000 & 0.000 & 0.000 & 0.100 \\
\hline 12057 & Técpan de Galeana & 0.000 & 0.200 & 0.000 & 0.000 & 0.000 \\
\hline 13006 & Alfajayucan & 4.600 & 16.100 & 10.900 & 1.600 & 9.300 \\
\hline 13061 & Tepeapulco & 5.500 & 16.900 & 2.500 & 0.100 & 0.300 \\
\hline 13063 & Tepeii del Río de Ocampo & 0.000 & 0.100 & 0.100 & 0.000 & 0.100 \\
\hline 14093 & Tepatitlán de Morelos & 0.000 & 0.400 & 0.100 & 0.000 & 0.000 \\
\hline 15090 & Tenango del Valle & 0.000 & 6.500 & 6.700 & 4.400 & 2.200 \\
\hline 15113 & Villa Guerrero & 0.000 & 0.000 & 0.000 & 0.000 & 0.000 \\
\hline 16076 & Sahuayo & 0.000 & 0.000 & 0.000 & 0.000 & 0.100 \\
\hline 16079 & Salvador Escalante & 1.300 & 0.400 & 0.000 & 0.300 & 0.000 \\
\hline 16102 & Uruapan & 0.200 & 0.000 & 0.000 & 0.100 & 0.000 \\
\hline 16107 & Zacapu & 0.000 & 0.000 & 0.000 & 0.000 & 0.000 \\
\hline 19004 & Allende & 0.100 & 0.100 & 0.100 & 0.000 & 0.000 \\
\hline 19033 & Linares & 0.800 & 0.500 & 1.800 & 2.100 & 4.700 \\
\hline 19044 & Sabinas Hidalgo & 1.000 & 1.100 & 6.100 & 6.500 & 3.300 \\
\hline 20068 & Ocotlán de Morelos & 5.500 & 15.600 & 2.200 & 0.100 & 0.100 \\
\hline 21004 & Acatzingo & 1.500 & 3.700 & 1.200 & 1.000 & 0.800 \\
\hline 21164 & Tepeaca & 0.000 & 0.000 & 0.000 & 0.000 & 0.000 \\
\hline 21208 & Zacatlán & 0.100 & 0.100 & 0.000 & 0.000 & 0.200 \\
\hline 22012 & Pedro Escobedo & 14.900 & 17.800 & 15.600 & 3.500 & 3.000 \\
\hline 22016 & San Juan del Río & 0.100 & 0.500 & 0.100 & 0.100 & 0.100 \\
\hline 25001 & Ahome & 0.000 & 0.000 & 0.300 & 0.700 & 0.700 \\
\hline 25006 & Culiacán & 0.000 & 0.000 & 0.000 & 0.000 & 0.000 \\
\hline 25012 & Mazatlán & 0.000 & 0.200 & 0.000 & 0.000 & 0.000 \\
\hline 26002 & Agua Prieta & 0.300 & 0.600 & 3.200 & 2.300 & 5.100 \\
\hline 26030 & Hermosillo & 1.400 & 4.700 & 6.600 & 1.500 & 4.600 \\
\hline 26043 & Nogales & 1.300 & 0.200 & 0.800 & 0.400 & 1.100 \\
\hline 30015 & Angel R. Cabada & 0.600 & 0.100 & 0.000 & 0.000 & 0.000 \\
\hline 30141 & San Andrés Tuxtla & 0.000 & 0.000 & 0.000 & 0.000 & 0.000 \\
\hline
\end{tabular}

Fuente: elaboración propia con base en datos de los censos económicos de los años incluidos. 
Cuadro A3. Eficiencia técnica de las unidades de análisis

\begin{tabular}{|c|c|c|c|c|c|c|}
\hline \multirow[t]{2}{*}{ Clave } & \multirow{2}{*}{$\begin{array}{l}\text { Zona Metropolitana / } \\
\text { municipio }\end{array}$} & \multicolumn{5}{|c|}{ Eficiencia } \\
\hline & & 1988 & 1993 & 1998 & 2003 & 2008 \\
\hline 1 & Aguascalientes & 0.69 & 0.93 & 0.94 & 0.92 & 0.96 \\
\hline 2 & Tïuana & 0.58 & 0.68 & 0.61 & 0.67 & 0.73 \\
\hline 3 & Mexicali & 0.58 & 0.86 & 0.88 & 0.82 & 0.85 \\
\hline 4 & La Laguna & 0.53 & 0.81 & 0.57 & 0.68 & 0.8 \\
\hline 5 & Saltillo & 0.98 & 0.96 & 0.95 & 0.95 & 0.96 \\
\hline 6 & Monclova-Frontera & 0.43 & 0.62 & 0.76 & 0.87 & 0.87 \\
\hline 8 & Colima-Villa de Álvarez & 0.34 & 0.36 & 0.2 & 0.67 & 0.26 \\
\hline 10 & Tuxtla Gutiérrez & 0.2 & 0.32 & 0.27 & 0.55 & 0.64 \\
\hline 11 & Juárez & $\cdot$ & 0.66 & 0.89 & 0.92 & 0.93 \\
\hline 12 & Chihuahua & $\cdot$ & 0.35 & 0.88 & 0.89 & 0.86 \\
\hline 13 & Valle de México & 0.46 & 0.67 & 0.11 & 0.25 & 0.23 \\
\hline 14 & León & - & 0.54 & 0.97 & 0.96 & 0.91 \\
\hline 18 & Pachuca & 0.65 & 0.83 & 0.35 & 0.2 & 0.39 \\
\hline 19 & Tulancingo & 0.41 & 0.36 & 0.56 & 0.21 & 0.23 \\
\hline 21 & Guadalajara & - & 0.78 & 0.36 & 0.46 & 0.46 \\
\hline 24 & Toluca & 0.9 & 0.95 & 0.9 & 0.9 & 0.86 \\
\hline 25 & Morelia & 0.19 & 0.3 & 0.2 & 0.21 & 0.22 \\
\hline 26 & Zamora-Jacona & 0.17 & 0.52 & 0.23 & 0.18 & 0.25 \\
\hline 28 & Cuernavaca & 0.97 & 0.97 & 0.92 & 0.86 & 0.85 \\
\hline 29 & Cuautla & - & 0.41 & 0.88 & 0.86 & 0.83 \\
\hline 30 & Tepic & 0.05 & 0.29 & 0.16 & 0.18 & 0.2 \\
\hline 31 & Monterrey & $\cdot$ & 0.7 & 0.71 & 0.75 & 0.72 \\
\hline 32 & Oaxaca & 0.13 & 0.74 & 0.15 & 0.17 & 0.07 \\
\hline 34 & Puebla-Tlaxcala & 0.84 & 0.8 & 0.92 & 0.95 & 0.95 \\
\hline 35 & Tehuacán & 0.43 & 0.51 & 0.24 & 0.16 & 0.26 \\
\hline 36 & Querétaro & 0.83 & 0.85 & 0.88 & 0.91 & 0.88 \\
\hline 38 & SLP-S. de Graciano Sánchez & 0.6 & 0.69 & 0.79 & 0.8 & 0.7 \\
\hline 43 & Reynosa-Río Bravo & - & 0.47 & 0.82 & 0.74 & 0.71 \\
\hline 44 & Matamoros & 0.9 & 0.87 & 0.87 & 0.93 & 0.92 \\
\hline
\end{tabular}


Eliseo Díaz, Jairo César López y Rafael Garduño

Cuadro A3. Eficiencia técnica de las unidades de análisis (continuación)

\begin{tabular}{|c|c|c|c|c|c|c|}
\hline \multirow[t]{2}{*}{ Clave } & \multirow{2}{*}{$\begin{array}{l}\text { Zona Metropolitana / } \\
\text { municipio }\end{array}$} & \multicolumn{5}{|c|}{ Eficiencia } \\
\hline & & 1988 & 1993 & 1998 & 2003 & 2008 \\
\hline 45 & Nuevo Laredo & 0.86 & 0.91 & 0.88 & 0.89 & 0.94 \\
\hline 46 & Tlaxcala-Apizaco & - & 0.46 & 0.57 & 0.46 & 0.44 \\
\hline 47 & Veracruz & - & 0.39 & 0.29 & 0.2 & 0.14 \\
\hline 49 & Poza Rica & - & 0.29 & 0.12 & 0.17 & 0.11 \\
\hline 50 & Orizaba & 0.15 & 0.1 & 0.15 & 0.17 & 0.06 \\
\hline 53 & Córdoba & - & 0.28 & 0.22 & 0.2 & 0.21 \\
\hline 54 & Acayucan & - & 0.24 & 0.19 & 0.26 & 0.22 \\
\hline 57 & Celaya & 0.87 & 0.78 & 0.88 & 0.75 & 0.79 \\
\hline 58 & Tianguistenco & 0.97 & 0.98 & 0.97 & 0.96 & 0.97 \\
\hline 2001 & Ensenada & - & 0.72 & 0.72 & 0.74 & 0.75 \\
\hline 5002 & Acuña & - & - & 0.96 & 0.97 & 0.96 \\
\hline 7019 & Comitán de Domínguez & - & 0.66 & 0.37 & 0.62 & 0.21 \\
\hline 8050 & Nuevo Casas Grandes & - & 0.73 & 0.94 & 0.98 & 0.95 \\
\hline 10005 & Durango & 0.26 & 0.17 & 0.41 & 0.1 & 0.45 \\
\hline 11017 & Irapuato & - & 0.27 & 0.44 & 0.25 & 0.17 \\
\hline 12057 & Técpan de Galeana & 0.17 & 0.72 & 0.31 & 0.45 & 0.06 \\
\hline 13006 & Alfajayucan & - & 0.98 & 0.97 & 0.65 & 0.96 \\
\hline 13061 & Tepeapulco & 0.94 & 0.98 & 0.84 & 0.44 & 0.38 \\
\hline 13063 & Tepeji del Río de Ocampo & 0.32 & 0.64 & 0.6 & - & 0.5 \\
\hline 14093 & Tepatitlán de Morelos & 0.22 & 0.8 & 0.62 & 0.34 & 0.39 \\
\hline 15090 & Tenango del Valle & - & 0.95 & 0.95 & 0.92 & 0.63 \\
\hline 15113 & Villa Guerrero & 0.03 & 0.26 & 0.24 & 0.16 & 0.29 \\
\hline 16076 & Sahuayo & - & 0.64 & 0.2 & 0.22 & 0.49 \\
\hline 16079 & Salvador Escalante & 0.79 & 0.55 & 0.2 & 0.38 & 0.2 \\
\hline 16102 & Uruapan & 0.66 & 0.35 & 0.14 & 0.44 & 0.19 \\
\hline 16107 & Zacapu & 0.05 & 0.36 & 0.26 & 0.29 & 0.32 \\
\hline 19004 & Allende & 0.31 & 0.63 & 0.61 & 0.62 & 0.54 \\
\hline 19033 & Linares & - & 0.64 & 0.84 & 0.89 & 0.94 \\
\hline 19044 & Sabinas Hidalgo & - & 0.83 & 0.95 & 0.96 & 0.91 \\
\hline
\end{tabular}


La eficiencia técnica de la industria automotriz en México, 1988-2008

\begin{tabular}{llrrrrr}
\hline Clave & Zona Metropolitana / & & & & & Eficiencia \\
\cline { 2 - 6 } & municipio & 1988 & 1993 & 1998 & 2003 & 2008 \\
\hline 20068 & Ocotlán de Morelos & 0.93 & 0.98 & 0.74 & 0.35 & 0.3 \\
21004 & Acatzingo & 0.65 & 0.9 & 0.72 & 0.67 & 0.47 \\
21164 & Tepeaca & 0.22 & 0.19 & 0.16 & 0.57 & 0.26 \\
21208 & Zacatlán & 0.21 & 0.31 & 0.28 & 0.15 & 0.75 \\
22012 & Pedro Escobedo & 0.98 & 0.98 & 0.98 & 0.91 & 0.89 \\
22016 & San Juan del Río & 0.41 & 0.76 & 0.38 & 0.59 & 0.44 \\
25001 & Ahome & - & 0.5 & 0.63 & 0.75 & 0.69 \\
25006 & Culiacán & 0.14 & 0.47 & 0.43 & 0.38 & 0.17 \\
25012 & Mazatlán & 0.23 & 0.79 & 0.34 & 0.27 & 0.2 \\
26002 & Agua Prieta & - & 0.91 & 0.92 & 0.89 & 0.94 \\
26030 & Hermosillo & 0.81 & 0.95 & 0.96 & 0.87 & 0.94 \\
26043 & Nogales & - & 0.84 & 0.84 & 0.83 & 0.79 \\
30015 & Angel R. Cabada & 0.41 & 0.3 & 0.15 & 0.51 & 0.03 \\
30141 & San Andrés Tuxtla & - & 0.18 & 0.13 & 0.15 & 0.07 \\
\hline
\end{tabular}

Fuente: elaboración propia con base en datos de los censos económicos de los años incluidos. 
